\title{
A necessary condition for incompatibility of observables in general probabilistic theories
}

\author{
Sergey N. Filippovt \\ Institute of Physics and Technology, Russian Academy of Sciences, Moscow, Russia and \\ Moscow Institute of Physics and Technology, Dolgoprudny, Moscow Region, Russia \\ Teiko Heinosaar $\left.\right|^{\dagger}$ and Leevi Leppäjärv用 \\ Turku Centre for Quantum Physics, Department of Physics and Astronomy, University of Turku, 20014, Finland
}

\begin{abstract}
We quantify the intrinsic noise content of an observable in a general probabilistic theory and derive a noise content inequality for incompatible observables. We apply the derived inequality to standard quantum theory, the quantum theory of processes, and polytope state spaces. The noise content for positive operator-valued measures takes a particularly simple form and equals the sum of minimal eigenvalues of all the effects. We illustrate our findings with a number of examples including the introduced notion of reverse observables.
\end{abstract}

PACS numbers: 03.65.Ta

\section{INTRODUCTION}

Quantum theory can be considered as a particular instance within a wide range of probabilistic theories [1, 2]. On the one hand, quantum theory inherits general properties of probabilistic theories and, consequently, one may deduce some features already from a general operational framework. For instance, the limitations on broadcastable subsets of states can be derived from this generality [3]. On the other hand, particular properties of quantum theory, like specific constraints on nonlocality, partially fix its position with respect to other probabilistic theories 4. As a result, specification of informationtheoretic axioms may be sufficient for quantum theory to be derived [5].

A general probabilistic theory operates with notions of states and observables. The set of states $\mathcal{S}$ is convex since any probabilistic mixture of states must be a valid state. Observables are then affine functionals from the set of states $\mathcal{S}$ to the set of probability distributions. In the standard quantum theory, states are associated with density operators, whereas observables are mathematically described by positive operator-valued measures (POVMs) 6 8. However, when we are testing a quantum process, then quantum channels are the examined objects and they are hence regarded as states, whereas observables can be described by process POVMs 911. Theories describing the Popescu-Rohrlich (PR) box 12 and polytope state spaces serve as other examples of general probabilistic theories [13, 14].

A set of observables in a general probabilistic theory may possess the property of being incompatible, which means that those observables cannot be seen as components of a single observable [15,18. Incompatibility is

\footnotetext{
sergey.filippov@phystech.edu

$\dagger$ teiko.heinosaari@utu.fi

$\ddagger$ leille@utu.fi
}

a non-classical feature, since in a general probabilistic theory with a classical state space all observables are compatible, while every non-classical theory possesses some incompatible observables [19, 20]. It is possible to compare the incompatibility of finite sets of observables in different probabilistic physical theories in a quantitative way [21 23 . Interestingly, quantum theory contains maximally incompatible pairs of observables, but only when the underlying Hilbert space is infinite dimensional 24.

This work focuses on incompatibility of observables in general probabilistic theories. The main goal of the present investigation is to quantify the noise content for observables in general probabilistic theories and to exploit it in deriving a sufficient condition for compatibility, i.e., a necessary condition for incompatibility for a collection of observables. To demonstrate that the derived condition is noteworthy, we use it to formulate a readily verifiable necessary condition for incompatibility in quantum theory. To anticipate this result, the condition takes the following form for POVMs: If $m$ POVMs are incompatible, then the sum of minimal eigenvalues of all their elements is less than $m-1$. We illustrate our findings by a number of examples including a newly introduced class of reverse observables. Consideration of standard quantum theory is followed by theories with quantum processes as states, as well as the square bit state space.

We note that in the case of POVMs, noise robustness of incompatibility has been investigated in several recent works 25 28]. The conditions found in those works are tighter than the condition presented in this work, but this is due to the fact that they are applicable only for POVMs with some specific structure or symmetry. Moreover, in contrast to most of the earlier studies (see for example [15, 17, 22, 29]), we do not add noise to given observables but rather look for the intrinsic noise which is already present. We show that a meaningful nontrivial noise inequality can be derived already at the level of a 
general probabilistic theory.

The paper is organized as follows. In Sec. II the incompatibility of observables in general probabilistic theories is reviewed. In Sec. III the noise content in observables is defined, and a sufficient condition for compatibility of a set of observables is formulated. The usage of the general condition is then demonstrated in Sec. IV.

\section{INCOMPATIBILITY OF OBSERVABLES IN GENERAL PROBABILISTIC THEORIES}

\section{A. States, effects, and observables}

We begin by recalling the basic elements of the standard framework of general probabilistic theories (see e.g. 30. 31 for more detailed presentations). In a general probabilistic theory, the set of states $\mathcal{S}$ is a convex subset of a finite dimensional real vector space $V$. The convexity is a result of the probabilistic nature of the theory, meaning that the convex sum $p s_{1}+(1-p) s_{2}$ is a state whenever $s_{1}, s_{2}$ are states and $0 \leq p \leq 1$.

We denote by $F(\mathcal{S})$ the linear space of all affine functionals from $\mathcal{S}$ to $\mathbb{R}$, i.e., a functional $e: \mathcal{S} \rightarrow \mathbb{R}$ is in $F(\mathcal{S})$ if it satisfies

$$
e\left(p s_{1}+(1-p) s_{2}\right)=p e\left(s_{1}\right)+(1-p) e\left(s_{2}\right)
$$

for all $s_{1}, s_{2} \in \mathcal{S}, 0 \leq p \leq 1$. For two functionals $e, f \in$ $F(\mathcal{S})$, we denote $e \leq f$ if $e(s) \leq f(s)$ for all $s \in \mathcal{S}$. We further denote by $u \in F(\mathcal{S})$ the unit map satisfying $u(s)=$ 1 for all $s \in \mathcal{S}$. The set of effects on $\mathcal{S}$ is defined as

$$
\mathcal{E}(\mathcal{S})=\{e \in F(\mathcal{S}): 0 \leq e \leq u\},
$$

i.e., it is the convex subset of those affine functionals $e$ for which $0 \leq e(s) \leq 1$ for all $s \in \mathcal{S}$. The set of effects arising as functionals on states is a particular example of an effect algebra 30 . In particular, $\mathcal{E}(\mathcal{S})$ has a partially defined sum $e+f$, which is simply the functional addition of $e$ and $f$ defined whenever $e+f \leq u$.

An observable with a finite number of outcomes is a function $\mathrm{A}: x \mapsto \mathrm{A}_{x}$ from a finite outcome set $X \subset \mathbb{Z}$ to $\mathcal{E}(\mathcal{S})$. The number $\mathrm{A}_{x}(s)$ is interpreted as the probability of getting the outcome $x$ in a measurement of the observable A when the system is in the state $s$. As we must have $\sum_{x \in X} \mathrm{~A}_{x}(s)=1$ for all $s \in \mathcal{S}$, we have the normalization condition $\sum_{x \in X} \mathrm{~A}_{x}=u$. We denote the set of observables with an outcome set $X$ by $\mathcal{O}_{X}$, and by $\mathcal{O}$ the set of all observables with a finite number of outcomes.

A special type of observable is the trivial observable $\mathrm{T}$, which is such that for each outcome $x, \mathrm{~T}_{x}(s)=\mathrm{T}_{x}\left(s^{\prime}\right)$ for all $s, s^{\prime} \in \mathcal{S}$. We denote the set of trivial observables by $\mathcal{T}$. Since the outcome probabilities for a trivial observable are the same for all states, it does not provide any information on an input state.

In what follows we recall the two most important instances of general probabilistic theories, standard quantum theory and the quantum theory of processes.
Example 1 (Quantum theory). Let $\mathcal{S}_{q}$ be the convex set of density operators $\varrho$ on a Hilbert space $\mathcal{H}$. Then the set of effects $\mathcal{E}\left(\mathcal{S}_{q}\right)$, defined as affine mappings on $\mathcal{S}_{q}$, can be represented as $e(\varrho)=\operatorname{tr}[\varrho E]$ for all states $\varrho$, where $E$ is a selfadjoint operator satisfying the operator inequalities $0 \leq E \leq \mathbb{1}$. This correspondence is one-to-one, so effects can be identified with these effect operators. With this identification, an observable $\mathrm{A}: x \mapsto \mathrm{A}_{x}$ with a finite outcome set $X$ is a POVM satisfying $\sum_{x \in X} \mathrm{~A}_{x}=\mathbb{1}$. A trivial observable $\mathrm{T}$ is of the form $\mathrm{T}_{x}=p_{x} \mathbb{1}$, where $p_{x}$ is a probability distribution on $X$.

Example 2 (Quantum theory of processes). We denote by $\mathcal{L}(\mathcal{H})$ the bounded linear operators on a Hilbert space $\mathcal{H}$. Let $\mathcal{S}_{p}$ be the set of completely positive and trace preserving maps $\Phi: \mathcal{L}\left(\mathcal{H}_{A}\right) \mapsto \mathcal{L}\left(\mathcal{H}_{B}\right)$, called quantum channels or processes. Then the set of effects $\mathcal{E}\left(\mathcal{S}_{p}\right)$ can be represented as the set of operators $M$ on $\mathcal{H}_{A} \otimes \mathcal{H}_{B}$ satisfying $0 \leq M \leq \varrho \otimes \mathbb{1}$ for some density operator $\varrho$ on $\mathcal{H}_{A}$. This representation is given as $e(\Phi)=\operatorname{tr}\left[\Omega_{\Phi} M\right]$, where $\Omega_{\Phi}$ is the Choi operator of $\Phi$, i.e., $\Omega_{\Phi}=(i d \otimes \Phi)\left[\left|\psi_{+}\right\rangle\left\langle\psi_{+}\right|\right]$, where $\psi_{+}=\sum_{i=1}^{d} \phi_{i} \otimes \phi_{i}$ and $\left\{\phi_{i}\right\}_{i=1}^{d}$ is an orthonormal basis of $\mathcal{H}_{A}$. An important point is that this correspondence between affine maps and operators is not one-toone; two operators $M$ and $M^{\prime}$ correspond to the same effect $e$ exactly when $M-M^{\prime}=\omega \otimes \mathbb{1}$ for some traceless operator $\omega[32$, 33. In this representation an observable $\mathrm{A}: x \mapsto \mathrm{A}_{x}$ with a finite outcome set $X$ satisfies the normalization $\sum_{x \in X} \mathrm{~A}_{x}=\varrho \otimes \mathbb{1}$ for some density operator $\varrho$ on $\mathcal{H}_{A}$. This kind of map is called a process POVM, or PPOVM for short [10. A trivial PPOVM is of the form $\mathrm{T}_{x}=p_{x} \xi_{x} \otimes \mathbb{1}$, where each $\xi_{x}$ is a density operator on $\mathcal{H}_{A}$ and $p_{x}$ is a probability distribution. Two trivial PPOVMs $\mathrm{T}_{x}=p_{x} \xi_{x} \otimes \mathbb{1}$ and $\mathrm{T}_{x}^{\prime}=p_{x}^{\prime} \xi_{x}^{\prime} \otimes \mathbb{1}$ correspond to the same trivial observable exactly when the probability distributions $p_{x}$ and $p_{x}^{\prime}$ are the same.

\section{B. Post-processing of observables}

A classical channel $\nu$ between outcome spaces $X$ and $Y$ is a right stochastic matrix with elements $\nu_{x y}, x \in X$, $y \in Y$, i.e., $0 \leq \nu_{x y} \leq 1$ and $\sum_{y \in Y} \nu_{x y}=1$. The number $\nu_{x y}$ is the transition probability for an element $x$ to be transformed into $y$. Classical channels are often used to describe noise, but we can also think of a classical channel as an active transformation that is implemented on outcomes. In the following we recall two classes of classical channels that will be used later.

Example 3 (Copying the measurement outcomes). Measurement outcomes are just classical symbols and thus can be copied. To see copying as a classical channel, let $Y=X \times X$. The stochastic matrix $\nu_{x y}^{\mathrm{c}}$ related to copying is defined as $\nu_{x y}^{\mathrm{c}}=1$ if $y=(x, x)$ and $\nu_{x y}^{\mathrm{c}}=0$ otherwise. This transforms any $x$ to $(x, x)$. Fig. 11(a) depicts the action of the copying channel. Multiple applications of a copying channel allows one to make an arbitrary number 
(a)

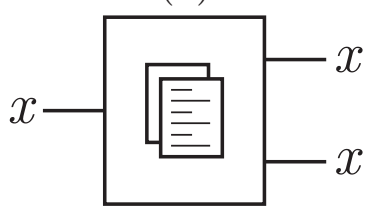

(b)

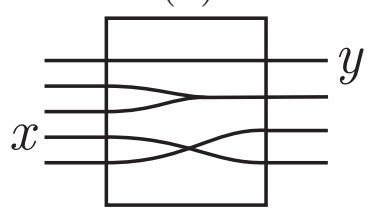

FIG. 1. (a) Action of the classical copying channel. Example of the relabeling channel.

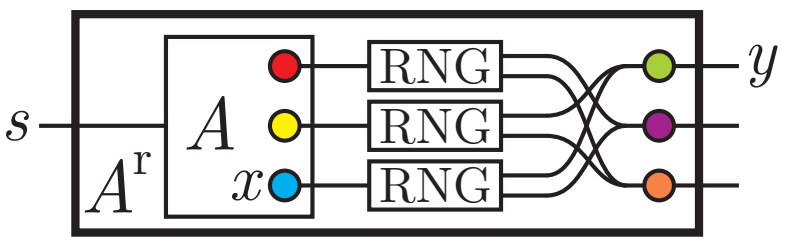

FIG. 2. $\quad A^{r}$ is the reverse observable with respect to $A$. Outcome $y$ of observable $A$ does not contribute to the outcome $y$ of observable $\mathrm{A}^{\mathrm{r}}$, so they are illustrated by complementary colors. RNG stands for a random number generator which uniformly chooses outcome $y \neq x$.

of copies of an outcome $x$. If the number of copies equals $m$, then we call it an $m$-copying channel.

Example 4 (Relabeling the measurement outcomes). The copying channels belong to a wider class of classical channels where measurement outcomes are relabeled deterministically into some other outcome. Let $f: X \rightarrow Y$ be a relabeling function. The derived stochastic matrix $\nu_{x y}^{f}$ is defined as $\nu_{x y}^{f}=1$ if $f(x)=y$ and $\nu_{x y}^{f}=0$ otherwise. In contrast to the copying procedure, generally several outcomes can be relabeled into a single new outcome, see Fig. 1(b).

Let $\mathrm{A}$ be an observable with an outcome set $X$ and let $\nu$ be a classical channel between $X$ and some other outcome space $Y$. We denote by $\nu \circ \mathrm{A}$ the new observable defined as

$$
(\nu \circ \mathrm{A})_{y}=\sum_{x \in X} \nu_{x y} \mathrm{~A}_{x}
$$

for all outcomes $y \in Y$. Physically, the observable $\nu \circ$ $A$ is implemented by first measuring $A$ and then using the classical channel $\nu$ on each obtained measurement outcome. This way of forming new observables gives rise to a preorder in the set of observables 34,36 . Namely, for two observables $\mathrm{A}$ and $\mathrm{B}$, we say that $\mathrm{B}$ is a postprocessing of $\mathrm{A}$ if there exists a classical channel $\nu$ such that $\mathrm{B}=\nu \circ \mathrm{A}$.

Example 5. (Reverse observable.) A reversing channel is a classical channel $\nu^{\mathrm{r}}: X \mapsto X$ such that $\nu_{x y}^{\mathrm{r}}=0$ if $x=y$ and $\nu_{x y}^{\mathrm{r}}=\nu_{x^{\prime} y}^{\mathrm{r}}$ for all $x, x^{\prime} \neq y$. If the outcome set $X$ contains $N$ elements, then $\nu_{x y}^{\mathrm{r}}=\nu_{x^{\prime} y}^{\mathrm{r}}=\frac{1}{N-1}$ for all $x, x^{\prime} \neq y$. For each observable $\mathrm{A}$, the observable $\mathrm{A}^{\mathrm{r}}=\nu^{\mathrm{r}} \circ \mathrm{A}$ is called the reverse version of $\mathrm{A}$. If A has $N$ outcomes, then the reverse observable $A^{r}$ takes the form

$$
\mathrm{A}_{x}^{\mathrm{r}}=\frac{1}{N-1} \sum_{y \neq x} \mathrm{~A}_{y}=\frac{1}{N-1}\left(u-\mathrm{A}_{x}\right) .
$$

The physical meaning of $A^{r}$ is illustrated in Fig. 2, After A has been measured and outcome $x$ has been obtained, we roll a fair dice with $N-1$ sides and randomly choose any outcome $y$ different from $x$. This is taken to be the outcome of the new observable $A^{r}$, which is hence given by formula 2 .

Example 6. (Doubly reverse observable.) Performing the reversing postprocessing two times, we get

$$
\mathrm{A}_{y}^{\mathrm{rr}}=\frac{1}{(N-1)^{2}}\left[\mathrm{~A}_{y}+(N-2) u\right]
$$

or, concisely, $\mathrm{A}^{\mathrm{rr}}=(1-\lambda) \mathrm{A}+\lambda \mathrm{T}$, where $\lambda=\frac{N(N-2)}{(N-1)^{2}}$ and $\mathrm{T}$ is the trivial observable with uniform distribution of outcomes. In the case of two outcomes $(N=2)$, the doubly reverse observable coincides with the original one, i.e., $A^{\mathrm{rr}}=A$.

As one would expect, a trivial observable $T$ is a postprocessing of any other observable $A$. To see this, we define a classical channel $\nu^{\top}$ as $\nu_{x y}^{\top}=\mathrm{T}_{y}\left(s_{0}\right)$ for all $x$, where $s_{0}$ is any state. Then

$$
\begin{aligned}
\left(\nu^{\top} \circ \mathrm{A}\right)_{y}(s) & =\sum_{x \in X} \nu_{x y}^{\top} \mathrm{A}_{x}(s)=\sum_{x \in X} \mathrm{~T}_{y}\left(s_{0}\right) \mathrm{A}_{x}(s)=\mathrm{T}_{y}\left(s_{0}\right) \\
& =\mathrm{T}_{y}(s),
\end{aligned}
$$

showing that $\nu^{\top} \circ \mathrm{A}=\mathrm{T}$. The classical channel $\nu^{\top}$ just erases the outcome obtained in the A-measurement, and replaces it with a new outcome according to the measurement outcome distribution of $\mathrm{T}$, which is the same for all states.

\section{Incompatibility of observables}

A collection of observables $\mathcal{P}$ is compatible if there exists an observable $\mathrm{C}$, with an outcome set $Y$, such that each observable $A \in \mathcal{P}$ is a post-processing of $C$. A compatible collection of observables can thus be implemented simultaneously by first measuring $\mathrm{C}$, then copying the classical outcomes, and finally applying the relevant postprocessings to the copied outcomes. This definition is depicted in Fig. 3. If a set of observables is not compatible, then it is called incompatible.

Let $\left\{\mathrm{A}^{(1)}, \ldots, \mathrm{A}^{(m)}\right\}$ be a compatible set of $m$ observables, with outcome sets $X^{(1)}, \ldots, X^{(m)}$, respectively. Thus, there exists an observable $C$ and classical channels $\nu^{(1)}, \ldots, \nu^{(m)}$ such that

$$
\mathrm{A}^{(j)}=\nu^{(j)} \circ \mathrm{C}, \quad j=1, \ldots, m .
$$




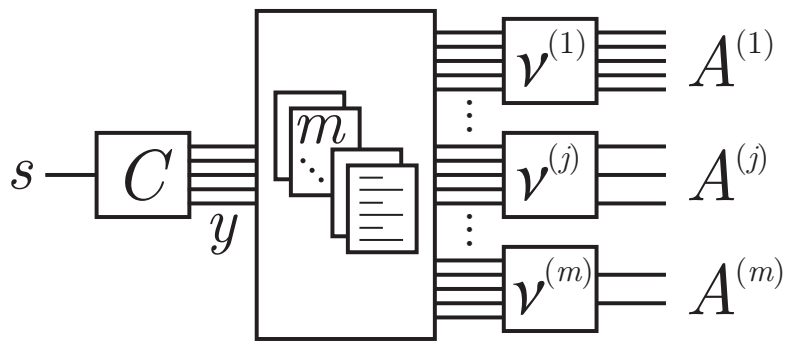

FIG. 3. Observables $A^{(1)}, \ldots, A^{(m)}$ are compatible if each of them is a post-processing of some observable $C$.

To see this definition of compatibility in an equivalent form, we denote

$$
\mathrm{G}_{x^{(1)} \ldots x^{(m)}}=\sum_{y} \prod_{j=1}^{m} \nu_{y x^{(j)}}^{(j)} \mathrm{C}_{y}
$$

for all $x^{(j)} \in X^{(j)}, j=1, \ldots, m$. Then $\mathrm{G}$ is an observable, and from (4) it follows that

$$
\mathrm{A}_{x^{(j)}}^{(j)}=\sum_{x^{(i)}: i \neq j} \mathrm{G}_{x^{(1)} \ldots x^{(m)}}
$$

Thus, the compatibility of observables $A^{(1)}, \ldots, A^{(m)}$ implies that there exists a joint observable $\mathrm{G}$ with the outcome space $X^{(1)} \times \cdots \times X^{(m)}$ such that the observables are marginals of the joint observable. Conversely, starting from $\mathrm{G}$ and taking classical channels corresponding to relabeling functions that are projections, $p r_{\ell}: X^{n} \rightarrow X$, $\operatorname{pr}_{\ell}\left(x_{1}, \ldots, x_{n}\right)=x_{\ell}$, we see that (6) is a special case of (4). As noted in 37 in the case of quantum observables, we conclude that a subset of observables is compatible if and only if they have a joint observable. The latter condition is usually taken as the definition of joint measurability of quantum observables 38.

\section{NECESSARY CONDITION FOR INCOMPATIBILITY}

\section{A. Noise content of an observable}

In order to formulate a necessary condition for incompatibility of observables, we first quantify their intrinsic fuzziness, or noise content, and then use the extraction of that noise in an explicit construction of a class of joint observables.

In a general probabilistic theory, one can introduce a procedure of mixing observables. Suppose $A: X \rightarrow \mathcal{E}(\mathcal{S})$ and $\mathrm{B}: Y \rightarrow \mathcal{E}(\mathcal{S})$ are observables with outcome sets $X$ and $Y$, respectively. Then a mixture of $\mathrm{A}$ and $\mathrm{B}$, with a mixing parameter $0 \leq t \leq 1$, is an observable $\mathrm{C}: X \cup Y \rightarrow$ $\mathcal{E}(\mathcal{S})$ such that

$$
\mathrm{C}_{z}=t \mathrm{~A}_{z}+(1-t) \mathrm{B}_{z}
$$

for all $z \in X \cup Y$, where $\mathrm{A}$ and $\mathrm{B}$ can be extended to $X \cup Y$ by defining $\mathrm{A}_{z}=0$ if $z \notin X$ and $\mathrm{B}_{z}=0$ if $z \notin Y$.

We are interested in a situation where one of the observables in the right-hand side of mixture 77 is not arbitrary but belongs to a some specified subset $\mathcal{N} \subseteq \mathcal{O}$ which describes noise in the measurement. If the target observable $\mathrm{C}$ is not in $\mathcal{N}$, then this requirement imposes limitations on possible values of the mixing parameter $t$.

For the following consideration, we fix a nonempty subset $\mathcal{N} \subseteq \mathcal{O}$ which describes noisy observables. Then, the physical meaning of Eq. (7) is to decompose an observable into its noisy part and the rest. A quantitative description of the noise content is attained by maximizing $t$. Therefore, for each observable $A$, we denote

$$
\begin{array}{r}
w(\mathrm{~A} ; \mathcal{N})=\sup \{0 \leq t \leq 1: t \mathrm{~N}+(1-t) \mathrm{B}=\mathrm{A} \\
\text { for some } \mathrm{N} \in \mathcal{N} \text { and } \mathrm{B} \in \mathcal{O}\}
\end{array}
$$

and call this quantity the noise content of $\mathrm{A}$ with respect to $\mathcal{N}$. We note that the observables $\mathrm{N}$ and $\mathrm{B}$ in $(8)$ can be assumed to have the same outcome set as $A$.

Whenever $\mathrm{A}_{x} \geq t \mathrm{~N}_{x}$ for some $0 \leq t \leq 1$ for all $x \in X$, we will use the notation $A \stackrel{t}{\geq} N$. Suppose $0 \leq t<1$ and $A \stackrel{t}{\geq} N$, then we can write $A$ as a mixture

$$
\mathrm{A}=t \mathrm{~N}+(1-t) \widetilde{\mathrm{A}}
$$

where $\widetilde{A}$ is the observable defined as

$$
\widetilde{\mathrm{A}}=(1-t)^{-1}(\mathrm{~A}-t \mathrm{~N}) \text {. }
$$

Conversely, if there exists some observable $\widetilde{A}$ such that (9) holds, then $A \stackrel{t}{\geq} \mathrm{N}$. Thus, one can reformulate the definition of noise content of $A$ with respect to $\mathcal{N}$ as follows:

$$
w(\mathrm{~A} ; \mathcal{N})=\sup \{0 \leq t \leq 1: \mathrm{A} \stackrel{t}{\geq} \mathrm{N} \text { for some } \mathrm{N} \in \mathcal{N}\}
$$

Specific properties of the map $\mathrm{A} \mapsto w(\mathrm{~A} ; \mathcal{N})$ depend on the choice of the subset $\mathcal{N}$. There are, however, some general features valid for any noise set $\mathcal{N}$. In particular, we observe the following:

(a) If $\nu$ is a classical channel and $\nu \circ \mathcal{N} \subseteq \mathcal{N}$, then $w(\nu \circ \mathrm{A} ; \mathcal{N}) \geq w(\mathrm{~A} ; \mathcal{N})$.

(b) If $\mathcal{N}$ is convex, then $w(s \mathrm{~A}+(1-s) \mathrm{B} ; \mathcal{N}) \geq$ $s w(\mathrm{~A} ; \mathcal{N})+(1-s) w(\mathrm{~B} ; \mathcal{N})$ for all $0 \leq s \leq 1$.

The first property follows directly from the definition of $w(\mathrm{~A} ; \mathcal{N})$, while the latter is seen to be valid by first noticing that

$$
s \mathrm{~A}_{x}+(1-s) \mathrm{B}_{x} \geq s w(\mathrm{~A} ; \mathcal{N}) \mathrm{N}_{x}+(1-s) w(\mathrm{~B} ; \mathcal{N}) \mathrm{M}_{x}
$$

for some observables $\mathrm{N}, \mathrm{M} \in \mathcal{N}$ and all outcomes $x$. We denote

$$
\begin{aligned}
& p_{\mathrm{A}}=s w(\mathrm{~A} ; \mathcal{N}) /[s w(\mathrm{~A} ; \mathcal{N})+(1-s) w(\mathrm{~B} ; \mathcal{N})] \\
& p_{\mathrm{B}}=(1-s) w(\mathrm{~B} ; \mathcal{N}) /[s w(\mathrm{~A} ; \mathcal{N})+(1-s) w(\mathrm{~B} ; \mathcal{N})]
\end{aligned}
$$


and then obtain

$s \mathrm{~A}_{x}+(1-s) \mathrm{B}_{x} \geq[s w(\mathrm{~A} ; \mathcal{N})+(1-s) w(\mathrm{~B} ; \mathcal{N})]\left(p_{\mathrm{A}} \mathrm{N}+p_{\mathrm{B}} \mathrm{M}\right)_{x}$,

where $p_{\mathrm{A}} \mathrm{N}+p_{\mathrm{B}} \mathrm{M} \in \mathcal{N}$ as the set $\mathcal{N}$ is convex.

The prototypical choice for $\mathcal{N}$ is to take $\mathcal{N}=\mathcal{T}$, the set of all trivial observables. In this case, we simply say that $w(\mathrm{~A} ; \mathcal{T})$ is the noise content of $\mathrm{A}$. The set $\mathcal{T}$ is convex and $\nu \circ \mathcal{T} \subseteq \mathcal{T}$ for all classical channels.

Proposition 1. Let $\mathrm{A}$ be an observable on a finite outcome set $X$. Then $w(\mathrm{~A} ; \mathcal{T})=\sum_{x \in X} \inf _{s \in \mathcal{S}} \mathrm{A}_{x}(s)$.

Proof. Denote $a_{x}=\inf _{s \in \mathcal{S}} \mathrm{A}_{x}(s)$ and $a=\sum_{x} a_{x}$. First assume that $a_{x}=0$ for all $x \in X$ so that $a=0$. Let $\mathrm{T} \in \mathcal{T}$ be a trivial observable and take any $t, 0 \leq t \leq 1$, such that $\mathrm{A} \stackrel{t}{\geq} \mathrm{T}$. By our definitions this is equivalent to $\mathrm{A}_{x}(s) \geq t \mathrm{\top}_{x}(s)$ for all $x \in X$ and $s \in \mathcal{S}$, so that for all $x \in X$ we have that

$$
0=a_{x}=\inf _{s \in \mathcal{S}} \mathrm{A}_{x}(s) \geq t \inf _{s \in \mathcal{S}} \mathrm{T}_{x}(s)=t p_{x},
$$

where $p_{x} \equiv \mathrm{T}_{x}(s)$ is the probability distribution defined by T. Summing over $x$ we get

$$
0=a=\sum_{x} a_{x} \geq t \sum_{x} p_{x}=t .
$$

Since also $0 \leq t \leq 1$, we must have $t=0$, and since this holds for all $\mathrm{T} \in \mathcal{T}$, by 11 we get that $w(\mathrm{~A} ; \mathcal{T})=a=0$.

Secondly, assume that $a_{x} \neq 0$ at least for some $x \in X$. By similar arguments as above, we see that for all $x \in X$ we have $a_{x} \geq t^{\prime} p_{x}^{\prime}$, where $p_{x}^{\prime}=\mathrm{T}_{x}^{\prime}(s)$ is a probability distribution defined by some trivial observable $\mathrm{T}^{\prime} \in \mathcal{T}$ for some $0 \leq t^{\prime} \leq 1$. Summing over all $x$ we then get an upper bound for $t^{\prime}$ as $a=\sum_{x} a_{x} \geq t^{\prime}$. We see that the upper bound is attained if we define $\mathrm{T}^{\prime}$ as $\mathrm{T}^{\prime}(s)=p_{x}^{\prime}=a_{x} / a$. Thus by 11 we have that $w(\mathrm{~A} ; \mathcal{T})=a$.

\section{B. Joint measurement scheme}

The joint measurement scheme that we will next discuss is an elaboration of the one presented in 28. The idea is that we first write the definition of compatibility in a slightly different way, then limit the defining conditions, and in this way we obtain a computable sufficient condition for compatibility.

From the definition, two observables A and B are compatible if there exists a third observable $C$ and classical channels $\nu_{1}$ and $\nu_{2}$ such that $\mathrm{A}=\nu_{1} \circ \mathrm{C}$ and $\mathrm{B}=\nu_{2} \circ \mathrm{C}$. Let us consider a seemingly more general scheme, where we are asking for the existence of two observables $\mathrm{C}$ and $\mathrm{D}$, classical channels $\nu_{1}, \nu_{2}, \mu_{1}$ and $\mu_{2}$, and a mixing parameter $t$ such that

$$
\begin{aligned}
& \mathrm{A}=t \nu_{1} \circ \mathrm{C}+(1-t) \mu_{1} \circ \mathrm{D} \\
& \mathrm{B}=t \nu_{2} \circ \mathrm{C}+(1-t) \mu_{2} \circ \mathrm{D} .
\end{aligned}
$$

Thus, $A$ and $B$ are now required to be mixtures of postprocessings of $C$ and D; see Fig. 4 .

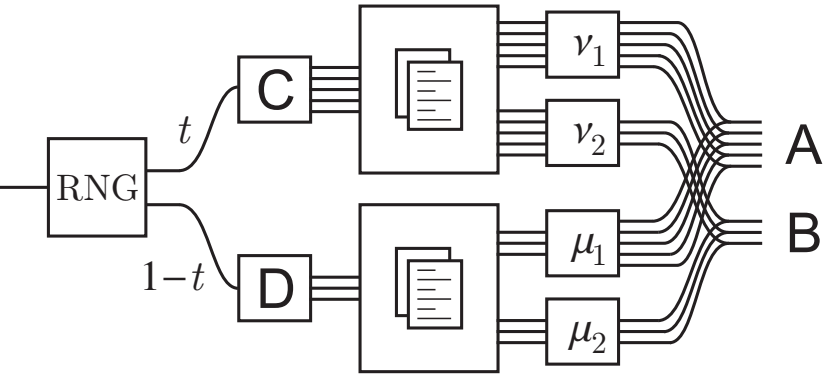

FIG. 4. The considered joint measurement scheme for two observables $A$ and $B$ consists of a random choice between two observables $C$ and $D$, followed by separated post-processing for both $A$ and $B$ that aim to approximate these observables.

Clearly, the conditions (12)-13 reduce to the usual compatibility conditions when $t=1$. Therefore, every compatible pair can be written in this new form. Conversely, if two observables $A$ and $B$ can be written in the form 12 - 13), then they are compatible. In fact, $A$ and $B$ are post-processings of the mixed observable $t C+(1-t) D$, but now the mixture has an extra outcome to keep track of which observable was measured each time. After measuring either C or D, we duplicate the outcome and post-process with either $\nu_{1}$ and $\nu_{2}$ or $\mu_{1}$ and $\mu_{2}$, depending on the measured observable.

\section{Incompatibility inequality}

As a special case of the joint measurement scheme described previously, we limit the choice of classical channels $\mu_{1}$ and $\nu_{2}$ to those that make observables $\mu_{1} \circ \mathrm{D}$ and $\nu_{2} \circ \mathrm{C}$ trivial. Since any trivial observable is a postprocessing of any other observable, we get all trivial observables, irrespective of $C$ and D. Hence, the conditions (12) - 13 reduce to

$$
\begin{aligned}
& \mathrm{A}=t \nu_{1} \circ \mathrm{C}+(1-t) \mathrm{T}_{1} \\
& \mathrm{~B}=t \mathrm{~T}_{2}+(1-t) \mu_{2} \circ \mathrm{D},
\end{aligned}
$$

where $T_{1}$ and $T_{2}$ are arbitrary trivial observables. Since we have added an extra limitation to the conditions $(12)$ (13), we cannot be sure anymore that a pair of compatible observables have this kind of representation. However, if $w(\mathrm{~A} ; \mathcal{T}) \geq 1-t$ and $w(\mathrm{~B} ; \mathcal{T}) \geq t$, then by the definition of noise content we can find suitable observables $C$ and $D$ such that (14)-15 hold.

As a conclusion, we obtain the following result and its equivalent formulation.

Proposition 2. If $\mathrm{A}$ and $\mathrm{B}$ are two observables such that $w(\mathrm{~A} ; \mathcal{T})+w(\mathrm{~B} ; \mathcal{T}) \geq 1$, then they are compatible.

Proposition 3. If $\mathrm{A}$ and $\mathrm{B}$ are incompatible observables, then $w(\mathrm{~A} ; \mathcal{T})+w(\mathrm{~B} ; \mathcal{T})<1$.

The joint measurement scheme has a direct generalization for any finite number of observables. Let us consider 
$m$ observables $\mathrm{A}^{(1)}, \ldots, \mathrm{A}^{(m-1)}$ and $\mathrm{A}^{(m)}$. We can then generalize conditions $14-15$ to

$$
\mathrm{A}^{(j)}=p_{j} \nu_{j} \circ \mathrm{C}^{(j)}+\left(1-p_{j}\right) \mathrm{T}^{(j)},
$$

where $\mathbf{T}^{(j)}$ is an arbitraty trivial observable for each $j=$ $1, \ldots, m$ and $p_{j}$ is an arbitrary probability distribution. As above, if $w\left(\mathrm{~A}^{(j)} ; \mathcal{T}\right) \geq 1-p_{j}$ for all $j$ we can make (16) hold. By summing over $j$ we conclude the following generalization of Prop. 3 .

Proposition 4. If $\mathrm{A}^{(1)}, \mathrm{A}^{(2)}, \ldots, \mathrm{A}^{(m)}$ are incompatible observables, then $w\left(\mathrm{~A}^{(1)} ; \mathcal{T}\right)+\ldots+w\left(\mathrm{~A}^{(m)} ; \mathcal{T}\right)<m-1$.

\section{APPLICATIONS OF THE INCOMPATIBILITY CONDITION}

\section{A. Eigenvalue condition for POVMs}

If $A$ is an observable in finite dimensional quantum theory described by a POVM, we have that

$$
\inf _{s \in \mathcal{S}} \mathrm{A}_{x}(s)=\min _{\psi \neq 0} \frac{\left\langle\psi \mid \mathrm{A}_{x} \psi\right\rangle}{\langle\psi \mid \psi\rangle} .
$$

It follows that $\inf _{s \in \mathcal{S}} \mathrm{A}_{x}(s)$ is the smallest eigenvalue of the effect operator $A_{x}$. Hence, by Prop. 1 we conclude that $w(\mathrm{~A} ; \mathcal{T})$ is the sum of the minimal eigenvalues of operators $\mathrm{A}_{x}$. Combining this with Prop. 4 we reach the following necessary condition for incompatibility.

Corollary 1. If $\mathrm{A}^{(1)}, \ldots, \mathrm{A}^{(m)}$ is a collection of $m$ incompatible POVMs, then the sum of the minimal eigenvalues of all their effects is smaller than $m-1$.

We will next illustrate the use of Cor. 1 in the case of reverse observables. Consider a regular rank-1 POVM A, i.e., the effects of $\mathrm{A}$ read $\mathrm{A}_{x}=\frac{d}{N} P_{x}$, where $d$ is the dimension of the Hilbert space, $N$ is the number of outcomes and $P_{x}$ is a one-dimensional projection. Examples of regular rank-1 POVMs include all nondegenerate sharp POVMs and symmetric informationally complete POVMs.

As before, we denote by $\mathrm{A}^{\mathrm{r}}=\nu^{\mathrm{r}} \circ \mathrm{A}$ the reverse version of $A$. If $A$ is a regular rank- 1 POVM, then the smallest eigenvalue of each operator $\mathrm{A}_{x}^{\mathrm{r}}$ is $\frac{N-d}{N(N-1)}$. Applying Cor. 1. we conclude that the reverse versions of $m$ regular rank-1 POVMs with $N$ outcomes are compatible if

$$
N \geq(d-1) \cdot m+1 \text {. }
$$

It follows from this observation that, for instance, the reverse versions of two regular rank-1 POVMs in $d=2$ are compatible for all $N \geq 3$. One can readily find POVMs with two outcomes whose reverse versions are incompatible; this is the case whenever the original ones are incompatible since, in the case of two outcomes, reversing is a reversible classical channel. Since the reversing channel is more and more noisy when the number of outcomes increases, one may wonder if there are any incompatible collections of reverse POVMs when the number of outcomes is more than two. In the following example we present a triplet of regular rank-1 POVMs whose reverse versions are incompatible; the simple compatibility condition 18 is hence not trivial.

Example 7 (Incompatible reverse POVMs). Consider three orthonormal bases $\left\{\varphi_{i}\right\}_{i=1}^{3},\left\{\psi_{i}\right\}_{i=1}^{3}$, and $\left\{\chi_{i}\right\}_{i=1}^{3}$ in a three-dimensional Hilbert space $\mathcal{H}_{3}$ such that a set $\left\{\varphi_{i}, \psi_{j}, \chi_{k}\right\}$ is linearly independent for all fixed $i, j, k$. Let $\mathrm{A}, \mathrm{B}$ and $\mathrm{C}$ be the POVMs related to these bases, i.e., $\mathrm{A}_{i}=\left|\varphi_{i}\right\rangle\left\langle\varphi_{i}\left|, \mathrm{~B}_{i}=\right| \psi_{i}\right\rangle\left\langle\psi_{i}\right|$ and $\mathrm{C}_{i}=\left|\chi_{i}\right\rangle\left\langle\chi_{i}\right|$. The fact that the reverse POVMs $A^{r}, B^{r}, C^{r}$ are incompatible can be proven by a contradiction. Suppose $A^{r}, B^{r}, C^{r}$ are compatible, so that there exists a joint POVM G with elements $\mathrm{G}_{i j k}$ such that $\mathrm{A}_{i}^{\mathrm{r}}=\sum_{j k} \mathrm{G}_{i j k}, \mathrm{~B}_{j}^{\mathrm{r}}=\sum_{i k} \mathrm{G}_{i j k}$, and $\mathrm{C}_{k}^{\mathrm{r}}=\sum_{i j} \mathrm{G}_{i j k}$. As $\left\langle\varphi_{i} \mid \mathrm{A}_{i}^{\mathrm{r}} \varphi_{i}\right\rangle=0$ and all the operators $\mathrm{G}_{i j k}$ are positive, we have $\left\langle\varphi_{i} \mid \mathrm{G}_{i j k} \varphi_{i}\right\rangle=0$ and this further implies $\mathrm{G}_{i j k} \varphi_{i}=0$. Similarly, $\mathrm{G}_{i j k} \psi_{j}=0$ and $\mathrm{G}_{i j k} \chi_{k}=0$. Since the set $\left\{\varphi_{i}, \psi_{j}, \chi_{k}\right\}$ spans $\mathcal{H}_{3}$, we conclude that $\mathrm{G}_{i j k}=0$. This contradicts the normalization $\sum_{i j k} \mathrm{G}_{i j k}=\mathbb{1}$. Hence, the three POVMs $\mathrm{A}^{\mathrm{r}}, \mathrm{B}^{\mathrm{r}}, \mathrm{C}^{\mathrm{r}}$ are incompatible.

The sufficient condition (18) for compatibility of the reverse versions of regular rank-1 POVMs is not necessary. We will next demonstrate that there are compatible observables that do not satisfy (18).

Example 8 (Two mutually unbiased bases). Consider a $d$-dimensional Hilbert space $\mathcal{H}_{d}$ and an orthonormal basis $\left\{\varphi_{i}\right\}_{i=0}^{d-1}$ in it. We denote $\omega=e^{i 2 \pi / d}$ and define another orthonormal basis $\left\{\psi_{j}\right\}_{j=0}^{d-1}$ by

$$
\psi_{j}=\frac{1}{\sqrt{d}} \sum_{k=0}^{d-1} \omega^{j k} \varphi_{k} .
$$

These two bases are mutually unbiased, meaning that $\left|\left\langle\varphi_{i} \mid \psi_{j}\right\rangle\right|=\frac{1}{\sqrt{d}}$ for all $i, j=0, \ldots, d-1$. The related POVMs $\mathrm{A}_{i}=\left|\varphi_{i}\right\rangle\left\langle\varphi_{i}\right|$ and $\mathrm{B}_{j}=\left|\psi_{j}\right\rangle\left\langle\psi_{j}\right|$ consists of noncommuting projections and are hence incompatible.

The reverse versions $\mathrm{A}^{\mathrm{r}}$ and $\mathrm{B}^{\mathrm{r}}$ are incompatible if $d=$ 2, since then $A^{r}$ and $B^{r}$ are just relabelings of $A$ and B. However, for any $d \geq 3, \mathrm{~A}^{\mathrm{r}}$ and $\mathrm{B}^{\mathrm{r}}$ are compatible even if the inequality $(18)$ does not hold. To see this, we recall that by Prop. 2 in $39, \mathrm{~A}^{\mathrm{r}}$ and $\mathrm{B}^{\mathrm{r}}$ are compatible whenever there exists a quantum state $\sigma \in \mathcal{S}(\mathcal{H})$ such that

$$
\operatorname{tr}\left[\mathrm{A}_{i} \sigma\right]=\frac{1-\delta_{i 0}}{d-1} \quad \text { and } \operatorname{tr}\left[\mathrm{B}_{j} \sigma\right]=\frac{1-\delta_{j 0}}{d-1}
$$

It is not hard to check that the operator

$$
\begin{aligned}
\sigma= & \frac{1}{d-1} \sum_{i=1}^{d-1}\left|\varphi_{i}\right\rangle\left\langle\varphi_{i}\right| \\
& -\frac{1}{(d-1)(d-2)} \sum_{1 \leq i<j \leq d}\left(\left|\varphi_{i}\right\rangle\left\langle\varphi_{j}|+| \varphi_{j}\right\rangle\left\langle\varphi_{i}\right|\right)
\end{aligned}
$$

is a density operator and satisfies the conditions above. Therefore, $\mathrm{A}^{\mathrm{r}}$ and $\mathrm{B}^{\mathrm{r}}$ are compatible. 
As explained in Example 6, the reversing channel $\nu^{\mathrm{r}}$ can also be applied to an already reverse observable $A^{r}$ to obtain a doubly reverse observable $A^{\text {rr }}$. It is not hard to see from Prop. 1 1 that two doubly reverse observables are always compatible if their number of outcomes $N \geq 3$. More generally, a sufficient condition for compatibility of $m$ doubly reverse observables with $N$ outcomes each is $m \leq(N-1)^{2}$.

\section{B. Eigenvalue condition for PPOVMs}

Let $\mathrm{A}$ be a PPOVM with an outcome set $X$ and the normalization $\sum_{x \in X} \mathrm{~A}_{x}=\varrho \otimes \mathbb{1}$ for some state $\varrho$. We denote by $m_{x}$ the minimal eigenvalue of the PPOVM element $\mathrm{A}_{x}$ for each $x \in X$. The noise content of $\mathrm{A}$ satisfies

$$
w(\mathrm{~A} ; \mathcal{T}) \geq \sum_{x \in X} m_{x} .
$$

To see this, we define a trivial PPOVM T as

$$
\mathrm{T}_{x}=\frac{m_{x}}{m} \varrho \otimes \mathbb{1},
$$

where $m=\sum_{x \in X} m_{x}$. Since

$$
\mathrm{A}_{x} \geq m_{x} \mathbb{1} \otimes \mathbb{1} \geq m_{x} \varrho \otimes \mathbb{1},
$$

we can define

$$
\mathrm{A}_{x}^{\prime}=\frac{1}{1-m}\left(\mathrm{~A}_{x}-m_{x} \varrho \otimes \mathbb{1}\right)
$$

and $A^{\prime}$ is a valid PPOVM. We can then write

$$
\mathrm{A}=m \mathrm{~T}+(1-m) \mathrm{A}^{\prime},
$$

which confirms 21). Prop. 4 thus implies the following result, analogous to Cor. 1

Corollary 2. If $\mathrm{A}^{(1)}, \ldots, \mathrm{A}^{(m)}$ is a collection of $m$ incompatible PPOVMs, then the sum of the minimal eigenvalues of all their effects is smaller than $m-1$.

We note that in contrast to the case of POVMs, the eigenvalue formula 21 provides only a lower bound for the noise content of a PPOVM. For instance, let

$$
\mathrm{A}_{x}=p_{x}\left|\psi_{x}\right\rangle\left\langle\psi_{x}\right| \otimes \mathbb{1}
$$

where $\left\langle\psi_{x} \mid \psi_{y}\right\rangle=\delta_{x y}$ and $p_{x}$ is a probability distribution. Then $m_{x}=0$ for all $x$ and the right hand side of (21) equals 0 . But the PPOVM A is trivial, so that the left hand side of (21) equals 1.

\section{Polytope state spaces}

A compact convex subspace $P$ of a finite dimensional vector space $V$ is a polytope if it has a finite number of extreme elements. Let $\operatorname{ext}(P)=\left\{s_{1}, \ldots, s_{n}\right\}$ be the set

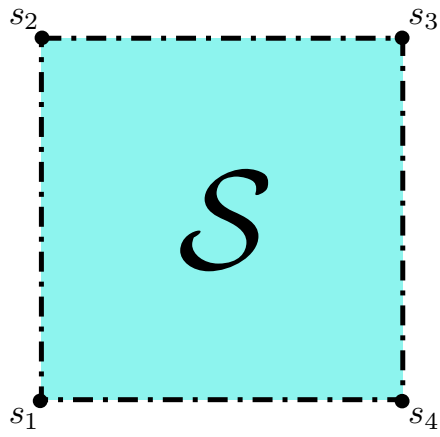

FIG. 5. Squit state space.

of extreme elements of a polytope $P$. Since every state $s \in P$ can be represented as a convex sum of elements in $\operatorname{ext}(P)$, we have that

$$
\begin{aligned}
\mathrm{A}_{x}(s) & =\mathrm{A}_{x}\left(\sum_{i} \lambda_{i} s_{i}\right)=\sum_{i} \lambda_{i} \mathrm{~A}_{x}\left(s_{i}\right) \\
& \geq \sum_{i} \lambda_{i} \min _{k} \mathrm{~A}_{x}\left(s_{k}\right)=\min _{k} \mathrm{~A}_{x}\left(s_{k}\right)
\end{aligned}
$$

for every $s \in P$, and thus inf $\operatorname{in}_{s \in \mathcal{S}} \mathrm{A}_{x}(s)=\min _{s \in \operatorname{ext}(P)} \mathrm{A}_{x}(s)$. Combining this with Prop. 4, we get an analogous result to the previous eigenvalue conditions for POVMs and PPOVMs.

Corollary 3. If $\mathrm{A}^{(1)}, \ldots, \mathrm{A}^{(m)}$ is a collection of $m$ incompatible observables on a polytopic state space $P$, then the sum of minimal values of all of their effects on $\operatorname{ext}(P)$ is smaller than $m-1$.

In the following, we take $\mathcal{S}$ to be a state space that is isomorphic to a square in $\mathbb{R}^{2}$, i.e., to the convex hull of four points $s_{1}, s_{2}, s_{3}, s_{4} \in \mathbb{R}^{2}$ satisfying $s_{1}+s_{3}=s_{2}+s_{4}$ (see Fig. 5). This is called the square bit state space, or squit state space for short.

We consider a class of binary observables $A^{\alpha}$ and $B^{\beta}$, parametrized by $\alpha, \beta \in[0,1]$, whose outcomes are labeled by \pm and defined on the extreme points $s_{1}, s_{2}, s_{3}$, and $s_{4}$ as

$$
\begin{array}{ll}
\mathrm{A}_{+}^{\alpha}\left(s_{1}\right)=\mathrm{A}_{+}^{\alpha}\left(s_{2}\right)=\alpha, & \mathrm{A}_{+}^{\alpha}\left(s_{3}\right)=\mathrm{A}_{+}^{\alpha}\left(s_{4}\right)=1, \\
\mathrm{~B}_{+}^{\beta}\left(s_{1}\right)=\mathrm{B}_{+}^{\beta}\left(s_{4}\right)=\beta, & \mathrm{B}_{+}^{\beta}\left(s_{2}\right)=\mathrm{B}_{+}^{\beta}\left(s_{3}\right)=1 .
\end{array}
$$

The values of $\mathrm{A}^{\alpha}$ and $\mathrm{B}^{\beta}$ are depicted in Fig. 6

Now we see that

$$
w\left(\mathrm{~A}^{\alpha} ; \mathcal{T}\right)=\min _{s \in \operatorname{ext}(\mathcal{S})} \mathrm{A}_{+}(s)+\min _{s \in \operatorname{ext}(\mathcal{S})} \mathrm{A}_{-}(s)=\alpha,
$$

and similarly that $w\left(\mathrm{~B}^{\beta} ; \mathcal{T}\right)=\beta$. Hence, by Cor. 3 , if

$$
\alpha+\beta \geq 1,
$$

then observables $A^{\alpha}$ and $B^{\beta}$ are compatible. It is easy to find $\mathrm{A}^{\alpha}$ and $\mathrm{B}^{\beta}$ as mixtures with maximal noise contents,

$$
\begin{aligned}
& \mathrm{A}^{\alpha}=\alpha \mathrm{T}+(1-\alpha) \mathrm{A} \\
& \mathrm{B}^{\beta}=\beta \mathrm{T}+(1-\beta) \mathrm{B},
\end{aligned}
$$



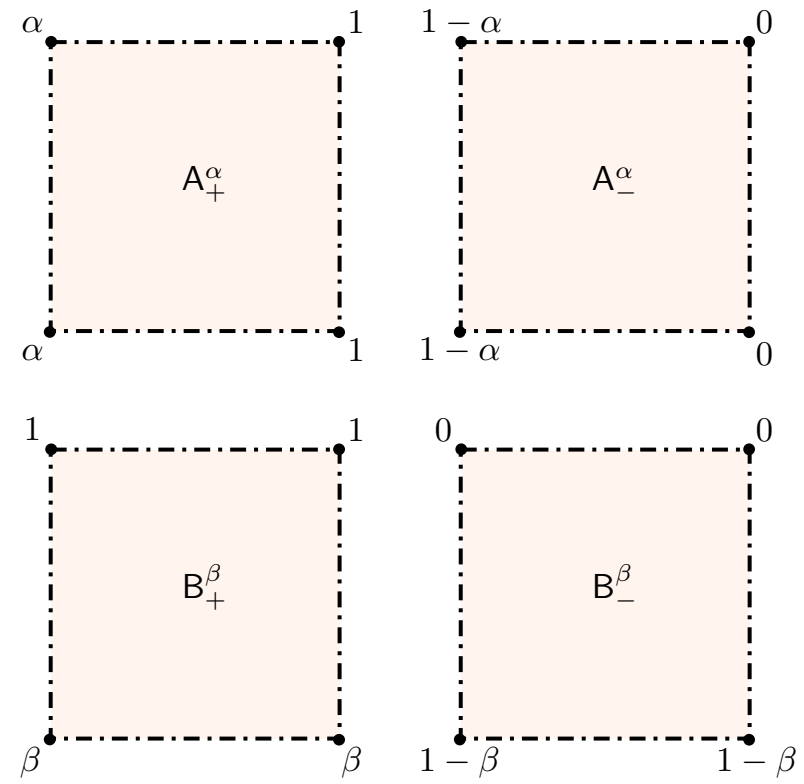

FIG. 6. Observables $\mathrm{A}^{\alpha}$ and $\mathrm{B}^{\beta}$.

where $\mathrm{T}$ is the trivial binary observable with $\mathrm{T}_{+}(s)=1$ and $\mathrm{T}_{-}(s)=0$ for all $s \in \mathcal{S}$, and $\mathrm{A} \equiv \mathrm{A}^{0}$ and $\mathrm{B} \equiv \mathrm{B}^{0}$.

The observables A and B are themselves incompatible. Moreso, they are maximally incompatible in the sense that the minimum amount of noise one has to mix them with to make their noisy versions compatible is enough to make any other pair of observables compatible. More precisely, it was shown in 21] that the observables $\lambda \mathrm{A}+$ $(1-\lambda) \mathrm{T}_{1}$ and $\mu \mathrm{B}+(1-\mu) \mathrm{T}_{2}$ are incompatible for all choices of trivial observables $T_{1}$ and $T_{2}$ if and only if $\lambda+\mu>1$. Therefore, we conclude that the inequality 27) derived from Prop. 2 is actually both necessary and sufficient for the compatibility of $\mathrm{A}^{\alpha}$ and $\mathrm{B}^{\beta}$.

\section{CONCLUSIONS}

We have considered general probabilistic theories on an equal footing and quantified the noise content of observables in every such theory via the set of trivial observables. In the case of standard quantum theory, the noise content is merely the sum of minimal eigenvalues of the POVM effects. In the quantum theory of processes, the noise content is bounded below by the sum of minimal eigenvalues of the corresponding PPOVM effects. In general, the noise content can be quantified with respect to any subset of observables.

We have derived the noise content inequality for a pair of observables, which is a necessary condition for their incompatibility. Our approach is based on a modification of the adaptive strategy for building a joint observable. We have then extended this result to the case of $m$ observables. By way of examples with reverse regular observables we have demonstrated non-triviality of the derived noise content inequality. Moreover, this inequality turned out to not only be necessary but also sufficient for incompatibility of some observables in the square bit state space.

\section{ACKNOWLEDGEMENTS}

The authors wish to thank Michal Sedlák and Mário Ziman for clarifying discussions concerning PPOVMs and Tom Bullock on useful comments on the manuscript. S.N.F. acknowledges the support of Academy of Finland for a mobility grant to conduct research in the University of Turku, where this article was initiated. S.N.F. is grateful to the University of Turku for kind hospitality. S.N.F. thanks the Russian Foundation for Basic Research for partial support under Project No. 16-37-60070 mola-dk.
[1] J. Barrett, Information processing in generalized probabilistic theories, Phys. Rev. A 75, 032304 (2007).

[2] G. Chiribella, G.M. D'Ariano, and P. Perinotti, Probabilistic theories with purification, Phys. Rev. A 81, 062348 (2010).

[3] H. Barnum, J. Barrett, M. Leifer, and A. Wilce, Generalized No-Broadcasting Theorem, Phys. Rev. Lett. 99, 240501 (2007).

[4] J. Allcock, N. Brunner, M. Pawlowski, V. Scarani, Recovering part of the boundary between quantum and nonquantum correlations from information causality, Phys. Rev. A 80, 040103(R) (2009).

[5] G. Chiribella and R.W. Spekkens (eds.), Quantum Theory: Informational Foundations and Foils, (Springer, 2016).
[6] P. Busch, M. Grabowski, and P. J. Lahti, Operational Quantum Physics, second corrected printing (SpringerVerlag, Berlin, 1997).

[7] A. S. Holevo, Statistical Structure of Quantum Theory (Springer-Verlag, Berlin, 2001).

[8] T. Heinosaari, M. Ziman, The mathematical language of quantum theory: From uncertainty to entanglement (Cambridge University Press, Cambridge, 2012).

[9] G. Chiribella, G. M. D'Ariano, and P. Perinotti, Memory Effects in Quantum Channel Discrimination, Phys. Rev. Lett. 101, 180501 (2008).

[10] M. Ziman, Process POVM: A mathematical framework for the description of process tomography experiments, Phys. Rev. A 77, 062112 (2008). 
[11] G. Chiribella, G. M. D'Ariano, and P. Perinotti, Theoretical framework for quantum networks, Phys. Rev. A 80, 022339 (2009)

[12] S. Popescu and D. Rohrlich, Nonlocality as an axiom, Found. Phys. 24, 379 (1994).

[13] G. Kimura, T. Miyadera, and H. Imai, Optimal state discrimination in general probabilistic theories, Phys. Rev. A 79, 062306 (2009).

[14] P. Janotta, C. Gogolin, J. Barrett, and N. Brunner, Limits on nonlocal correlations from the structure of the local state space, New J. Phys. 13, 063024 (2011).

[15] N. Stevens and P. Busch, Steering, incompatibility, and Bell-inequality violations in a class of probabilistic theories, Phys. Rev. A 89, 022123 (2014).

[16] M. Banik, Measurement incompatibility and Schrödinger-Einstein-Podolsky-Rosen steering in a class of probabilistic theories, J. Math. Phys. 56, 052101 (2015).

[17] T. Heinosaari, T. Miyadera, and M. Ziman, An invitation to quantum incompatibility, J. Phys. A: Math. Theor. 49, 123001 (2016)

[18] M. Sedlák, D. Reitzner, G. Chiribella and M. Ziman, Incompatible measurements on quantum causal networks, Phys. Rev. A 93, 052323 (2016).

[19] S. Aravinda, R. Srikanth, A. Pathak, On the origin of nonclassicality in single systems, arXiv:1607.01768 [quant-ph].

[20] M. Plávala, All measurements in a probabilistic theory are compatible if and only if the state space is a simplex, Phys. Rev. A 94, 042108 (2016).

[21] P. Busch, T. Heinosaari, J. Schultz, and N. Stevens, Comparing the degrees of incompatibility inherent in probabilistic physical theories, EPL 103, 10002 (2013).

[22] S. Gudder, Compatibility for probabilistic theories, arXiv:1303.3647 [quant-ph].

[23] M. Banik, Md. R. Gazi, S. Ghosh, and G. Kar, Degree of complementarity determines the nonlocality in quantum mechanics, Phys. Rev. A 87, 052125 (2013).

[24] T. Heinosaari, J. Schultz, A. Toigo, and M. Ziman, Maximally incompatible quantum observables, Phys. Lett. A 378, 1695 (2014).
[25] E. Haapasalo, Robustness of incompatibility for quantum devices, J. Phys. A: Math. Theor. 48, 255303 (2015).

[26] H. Zhu, Information complementarity: A new paradigm for decoding quantum incompatibility, Sci. Rep. 5, 14317 (2015).

[27] T. Heinosaari, J. Kiukas, and D. Reitzner, Noise robustness of the incompatibility of quantum measurements, Phys. Rev. A 92, 022115 (2015).

[28] R. Uola, K. Luoma, T. Moroder, and T. Heinosaari, Adaptive strategy for joint measurements, Phys. Rev. A 94, 022109 (2016).

[29] M. Wolf, D. Perez-Garcia, C. Fernandez, Measurements incompatible in quantum theory cannot be measured jointly in any other no-signaling theory, Phys. Rev. Lett. 103, 230402 (2009).

[30] E.G. Beltrametti and S. Bugajski, Effect algebras and statistical physical theories, J. Math. Phys. 38, 3020 (1997).

[31] H. Barnum and A. Wilce, Information Processing in Convex Operational Theories, Electronic Notes in Theoretical Computer Science 270:3-15 (2011).

[32] A. Jenčová, Generalized channels: Channels for convex subsets of the state space, J. Math. Phys. 53, 012201 (2012).

[33] A. Jenčová, Extremal generalized quantum measurements, Linear Algebra Appl. 439, 4070 (2013).

[34] H. Martens and W.M. de Muynck, Nonideal Quantum Measurements, Found. Phys. 20, 255 (1990).

[35] F. Buscemi, M. Keyl, G.M. D'Ariano, P. Perinotti, and R.F. Werner, Clean positive operator valued measures, J. Math. Phys. 46, 082109 (2005).

[36] T. Heinonen, Optimal measurements in quantum mechanics, Phys. Lett. A 346, 77 (2005).

[37] S.T. Ali, C. Carmeli, T. Heinosaari, and A. Toigo, Commutative POVMs and fuzzy observables, Found. Phys. 39, 593 (2009).

[38] P. Lahti, Coexistence and joint measurability in quantum mechanics, Int. J. Theor. Phys. 42, 893 (2003).

[39] C. Carmeli, T. Heinosaari, and A. Toigo, Informationally complete joint measurements on finite quantum systems, Phys. Rev. A 85, 012109 (2012) 